\title{
Physical And Experimental Background Of The Design Of The ELISE Test Facility
}

\author{
P. Franzen, U. Fantz, W. Kraus, H. Falter, B. Heinemann, \\ R. Nocentini and the NNBI Team
}

Max-Planck-Institut für Plasmaphysik, EURATOM Association, PO Box 1533, 85740 Garching, Germany

\begin{abstract}
In 2007 the IPP RF driven negative hydrogen ion source was chosen by the ITER board as the new reference source for the ITER neutral beam system. In order to support the design of the Neutral Beam Test Facility in Padua and its commissioning and operating phases, IPP is presently constructing a new test facility ELISE (Extraction from a Large Ion Source Experiment) for a large-scale extraction from a half-size ITER RF source. Plasma operation of up to one hour is foreseen; but due to the limits of the IPP HV system, pulsed extraction only is possible. The extraction system is designed for acceleration of negative ions of up to $60 \mathrm{kV}$. The start of the ELISE operation is planned for middle of 2010. The aim of the design of the ELISE source and extraction system was to be as close as possible to the ITER design; it has however some modifications allowing a better diagnostic access as well as more flexibility for exploring open questions. The design was also supported by diagnostics and modeling efforts of the processes leading to negative ion production and extraction in a RF source.
\end{abstract}

Keywords: Neutral Beam Injection, ITER, negative ion source, RF source

PACS: $28.52 . \mathrm{Cx}$, 52.27.Cm, 52.50.Dg, 52.50.Gj

\section{INTRODUCTION}

The development of large negative hydrogen ion sources for the ITER NBI system [1] was started in the early 90's in Japan with filamented arc sources as the basis for the design. Filamented sources, however, suffer from regular maintenance periods (twice per year in ITER) due to the limited lifetime of the filaments. Due to the advantages of the RF source - it is in principle maintenance-free - and due to the good experience with the positive ion based RF sources [2] at the NBI system for ASDEX Upgrade and W7-AS, IPP Garching started the development of a RF driven negative ion source end of the 90's, from 2002 on within a framework of an official EFDA contract. The development was very successful [3,4,5]: recently, in July 2007, the RF source was chosen by the ITER board as the reference source [6,7].

The development of the RF driven negative hydrogen ion source is being done at IPP at three test facilities in parallel: Current densities of $330 \mathrm{~A} / \mathrm{m}^{2}$ with $\mathrm{H}-$ and 230 $\mathrm{A} / \mathrm{m}^{2}$ with $\mathrm{D}-$ have been achieved with the IPP RF source on the small test facility BATMAN (Bavarian Test Machine for Negative Ions) at the required source pressure $(0.3 \mathrm{~Pa})$ and electron/ion ratios, but with a small extraction area $\left(70 \mathrm{~cm}^{2}\right)$ and limited pulse length $(<4 \mathrm{~s})$ [3]. The long pulse test facility MANITU (Multi Ampere Negative 


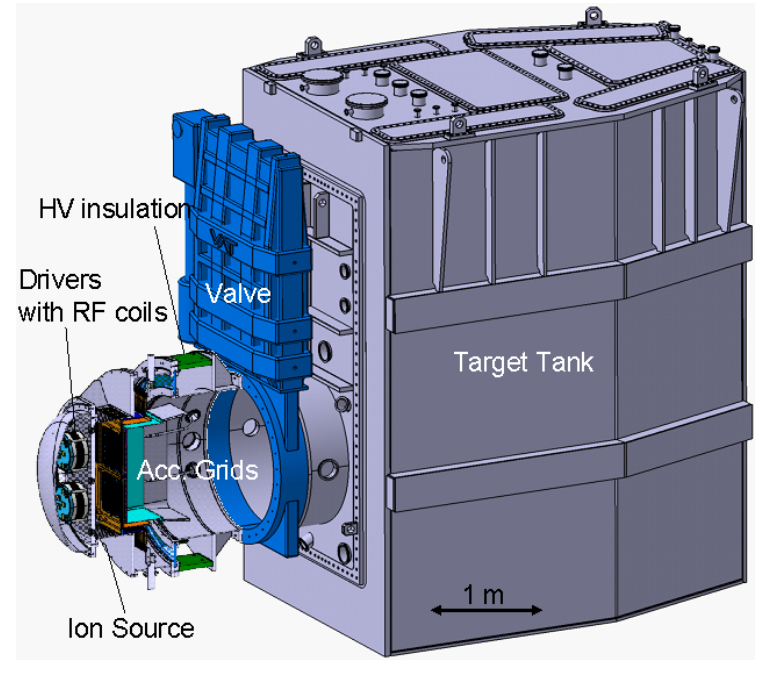

Figure 1. The ELISE test facility

Ion Test Unit) equipped with the same source as it is used at BATMAN but having an extraction area of about $200 \mathrm{~cm}^{2}$ demonstrated recently stable one hour pulses; the parameters however, are still below the ITER requirements $[8,9]$. The ion source test facility RADI, equipped with a source of approximately the width and half the height of the ITER source, aims to demonstrate the required plasma homogeneity of a large RF source [10,11,12]; its modular driver concept will allow a straightforward extrapolation to the full size ITER source.

The results of RADI, however, have limited significance due to the lack of extraction. Hence, IPP is presently designing a new test facility ELISE (Extraction from a Large Ion Source Experiment) for long pulse plasma operation and short pulse, but large-scale extraction from a half-size ITER source. ELISE is an important step between the small scale extraction experiments at BATMAN and MANITU and the full size ITER neutral beam system and supports the design of the Neutral Beam Test Facility in Padua and its commissioning and operating phases. The integrated commissioning of ELISE is planned for 2010, assuming a start of the project end of 2008.

Figure 1 shows an overview of the ELISE test facility. The detailed technical details are given in Refs. [13] and [14]. The aim of the design of the ELISE source and extraction system was to be as close as possible to the ITER design; it has however some modifications allowing a better diagnostic access as well as more experimental flexibility. The paper discusses these design choices and their physical and experimental background that was gained at the present IPP test facilities. Some of these design choices have been also already implemented in the design of the ITER ion source which is presently being finalized by RFX Padua [15].

\section{THE DESIGN OF ELISE}

Table 1 shows the main parameters of ELISE. Due to the limits of the IPP HV system, only pulsed extraction during a long plasma pulse is possible. How this will affect the performance of ELISE is not clear; experiments at MANITU showed that at least for a well conditioned source the same performance could be obtained during pulsed extraction during a long pulse [16].

The total maximum available voltage
TABLE 1. Parameters of the ELISE test facility.

\begin{tabular}{ll}
\hline Isotope & $\mathrm{H}, \mathrm{D}$ (limited) \\
Extraction Area & $1000 \mathrm{~cm}^{2}$ \\
Apertures & $640,2 \times 4$ groups, \\
& $14 \mathrm{~mm} \mathrm{Ø}$ \\
Source Size & $1.0 \times 0.873 \mathrm{~m}^{2}$ \\
Total Voltage & $<60 \mathrm{kV}$ \\
Extraction Voltage & $<12 \mathrm{kV}$ \\
Extracted Ion Current & $<25 \mathrm{~A} \mathrm{for} \mathrm{j}_{\mathrm{e}} / \mathrm{j}_{\mathrm{H}^{-}}=1$ \\
Acc. Ion Current & $<20 \mathrm{~A}$ \\
RF power & $<360 \mathrm{~kW}$ \\
Pulse Length: & \\
$\quad$ Plasma & $3600 \mathrm{~s}$ \\
$\quad$ Extraction & $10 \mathrm{~s}$ every $160 \mathrm{~s}$ \\
\hline
\end{tabular}


corresponds to the pre-acceleration voltage of the SINGAP accelerator design [17] (the MAMUG pre-acceleration voltage is larger, at $200 \mathrm{kV}$ ). The total available current is $50 \mathrm{~A}$. Taking the stripping losses of the ELISE grid system into account $(\sim 15 \%$, as measured in BATMAN with the SINGAPlike CEA grid system [18]), that current allows an operation at the required parameters with respect to the accelerated current density both in $\mathrm{H}$ and $\mathrm{D}\left(300 \mathrm{~A} / \mathrm{m}^{2}\right.$ and 200 $\mathrm{A} / \mathrm{m}^{2}$, respectively) at the respective electron/ion ratios ( 0.5 in $\mathrm{H}, 1.0$ in $\mathrm{D})$ as well as with respect to the required extracted current density in $\mathrm{H}\left(330 \mathrm{~A} / \mathrm{m}^{2}\right)$. But in order to achieve the required extracted ion current density in D $\left(290 \mathrm{~A} / \mathrm{m}^{2}\right)$, the amount of co-extracted electrons has to be further reduced to an electron/ion ratio of

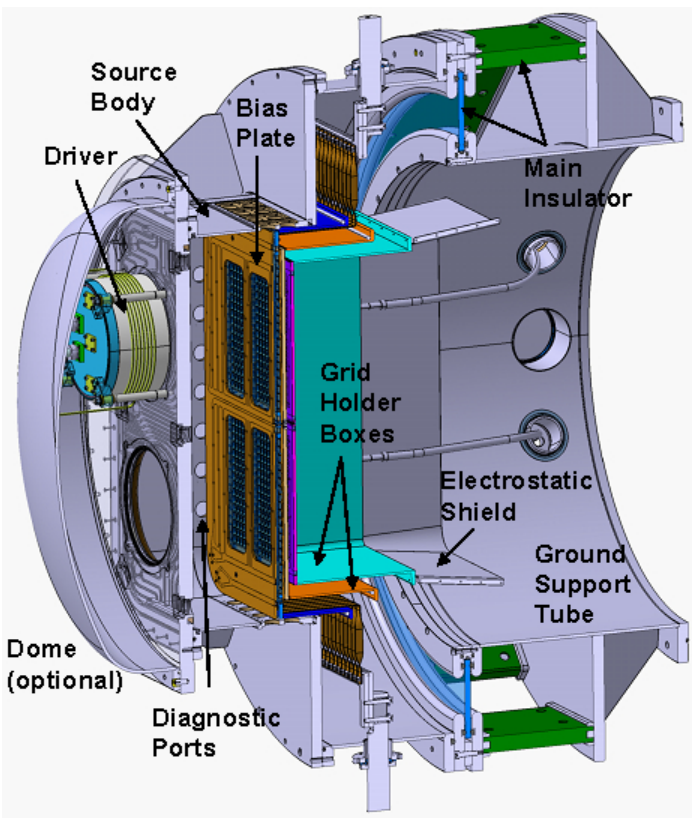

FIGURE 2. Details of the ELISE ion source and the extraction system about 0.8 .

Figure 2 shows the details of the ELISE ion source and the extraction system. In contrast to the ITER, the ELISE source will usually be operated in air - with a possibility to operate the drivers in vacuum - to facilitate diagnostic access near the most important region, i.e. the region near the plasma grid [12], and to provide experimental flexibility for e.g. an easy change of the magnetic field configuration. The necessary $\mathrm{HV}$ insulation of the source is done with a combination of a glass ring as vacuum boundary and epoxy plates for the mechanical support [13].

The experience at MANITU showed that a source valve is mandatory in order to keep the source performance - when it is cesiated - unaffected during the cryo pump recovery phase. At MANITU, the cryo pump is normally recovered during the night by the control system automatically. The Cs conditioning phase in the next morning was shorter when the pressure, at which the source valve is closed during the recovery phase, was reduced from $10^{-3}$ mbar to $10^{-5}$ mbar. This is a strong indication of a Cs poisoning effect of poor vacuum.

\section{Ion Source}

At the start of the activities, the design of the ELISE - and the ITER source - was based on the existing large RF source at RADI. However, Langmuir probe measurements showed that the electron density starts to decrease towards the source wall already within the projection of the driver (see Figure 3). Although there is not necessarily a strong correlation of the electron density

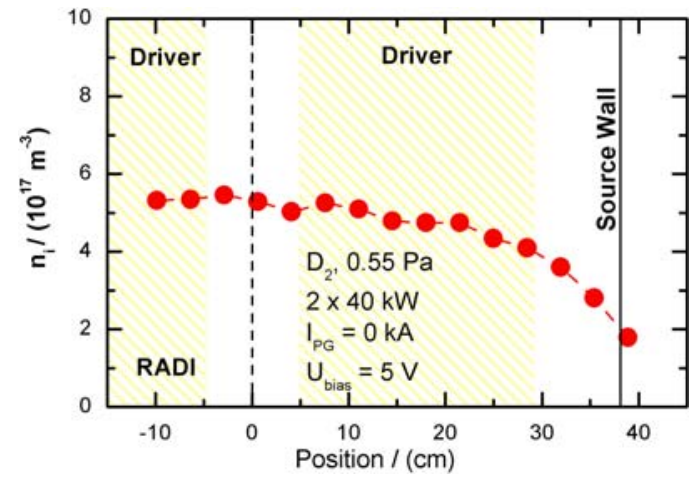

FIGURE 3. Plasma density profile in RADI in $2 \mathrm{~cm}$ distance from the (dummy) plasma grid. 
TABLE 2. Design power loads for the ELISE source components and the grids. The maximum total power levels are given for full performance operation $\left(\mathrm{P}_{\mathrm{RF}}=300 \mathrm{~kW}, 20 \mathrm{~A}\right.$ acc. current $)$.

\begin{tabular}{|c|c|c|c|c|}
\hline Component & $\begin{array}{l}\text { Amount of } \\
\text { RF power }\end{array}$ & $\begin{array}{c}\text { Max. Power } \\
\text { Density }\end{array}$ & Total Power & Comments \\
\hline Faraday Screens & $\sim 40 \%$ & $300 \mathrm{~kW} / \mathrm{m}^{2}$ & $120 \mathrm{~kW}$ & $\begin{array}{l}\text { measured at RADI and } \\
\text { BATMAN }\end{array}$ \\
\hline RF Coils & $\sim 5 \%$ & - & $15 \mathrm{~kW}$ & measured at RADI \\
\hline Bias Plate & $\sim 1 \%$ & $30 \mathrm{~kW} / \mathrm{m}^{2}$ & $3 \mathrm{~kW}$ & measured at MANITU \\
\hline Driver Back Plate: & & & & \\
\hline Eddy Currents & $2.5 \%$ & - & $7.5 \mathrm{~kW}$ & calculated \\
\hline Drivers Plate: & & & $67.5 \mathrm{~kW}$ & \\
\hline Eddy Currents & $2.5 \%$ & - & & calculated \\
\hline Driver Exit & $10 \%$ & $300 \mathrm{~kW} / \mathrm{m}^{2}$ & & estimated from FS power density \\
\hline Surface & $10 \%$ & $100 \mathrm{~kW} / \mathrm{m}^{2}$ & & estimated from power balance \\
\hline $\begin{array}{l}\text { Losses in RF lines } \\
\text { and Transformer }\end{array}$ & $\sim 5 \%$ & - & $15 \mathrm{~kW}$ & estimated \\
\hline Lateral Walls & $20 \%$ & $100 \mathrm{~kW} / \mathrm{m}^{2}$ & $60 \mathrm{~kW}$ & estimated from power balance \\
\hline Plasma Grid & $\sim 5 \%$ & $20 \mathrm{~kW} / \mathrm{m}^{2}$ & $20 \mathrm{~kW}$ & $\begin{array}{l}\text { measured at RADI (w/o bias } \\
\text { plate), including } 4 \mathrm{kA} \text { current }\end{array}$ \\
\hline Extraction Grid & - & $32 \mathrm{MW} / \mathrm{m}^{2}$ & $400 \mathrm{~kW}$ & $\begin{array}{l}\text { twice the nominal power density, } \\
\text { calculated with transport code }\end{array}$ \\
\hline Grounded Grid & - & & $60 \mathrm{~kW}$ & $\begin{array}{l}10 \% \text { of total beam power, } \\
\text { BATMAN experience }\end{array}$ \\
\hline
\end{tabular}

with the negative ion density for surface production, this decrease of the plasma density is expected to be not of advantage for a homogeneous beam. Hence, the size of the source for ELISE (and accordingly the size of the ITER source) was increased by about $100 \mathrm{~mm}$ in both directions having now $873 \mathrm{~mm}$ in width and $1000 \mathrm{~mm}$ in height.

Furthermore, the diameter of the drivers was increased from $245 \mathrm{~mm}$ to $284 \mathrm{~mm}$ (inside) and they were shifted radially outwards, so that the inner edges of the drivers are still at the same position. With this change, also the outermost apertures are within the drivers projection leading to a more homogeneous illumination of the grid.

Another still open point is the optimum depth of the ion source. The presently foreseen source depth is $238 \mathrm{~mm}$, as it is the case for the small IPP source; this is about a factor of 2 smaller than the filamented source. In order to have some experimental flexibility, the depth of the ELISE source can be enlarged by another $54 \mathrm{~mm}$ by a spacer ring between the source flange and the high voltage ring. An additional row of ports allows diagnostic access at the same position with respect to the plasma grid at the new position.

The expected power levels to the source components are shown in Table 2. As a full source calorimetry at RADI and MANITU is not available up to now (but it is part of the experimental program of the next months), the power loads of the drivers plate and the lateral walls are estimations from the missing power. As can be seen, the power density levels to the source components are rather low $\left(100-300 \mathrm{~kW} / \mathrm{m}^{2}\right)$.

For ELISE a cooled bias plate is foreseen with "window frame" like openings around the beamlet groups (see Figure 2). The windows are $12.5 \mathrm{~mm}$ larger than the outer edge of the apertures in all directions. The bias plate is insulated against the source body so that the potential of the plate can be changed with respect to the body 
- and the plasma grid - as it is the case now at MANITU. First experiments here showed that the electron current could be reduced by changing the bias plate potential [16].

The installation of a bias plate enhanced the extracted negative ion current significantly on the test facility BATMAN. In this test facility the bias plate is electrically connected to the source body potential and extends that potential to the edge of the apertures. The use of this bias plate reduced the necessary filter field strength for suppression of the co-extracted electrons. The latter was not a big problem for hydrogen operation, but was essential for deuterium operation [19].

For the present experimental period, almost all inner copper surfaces of the

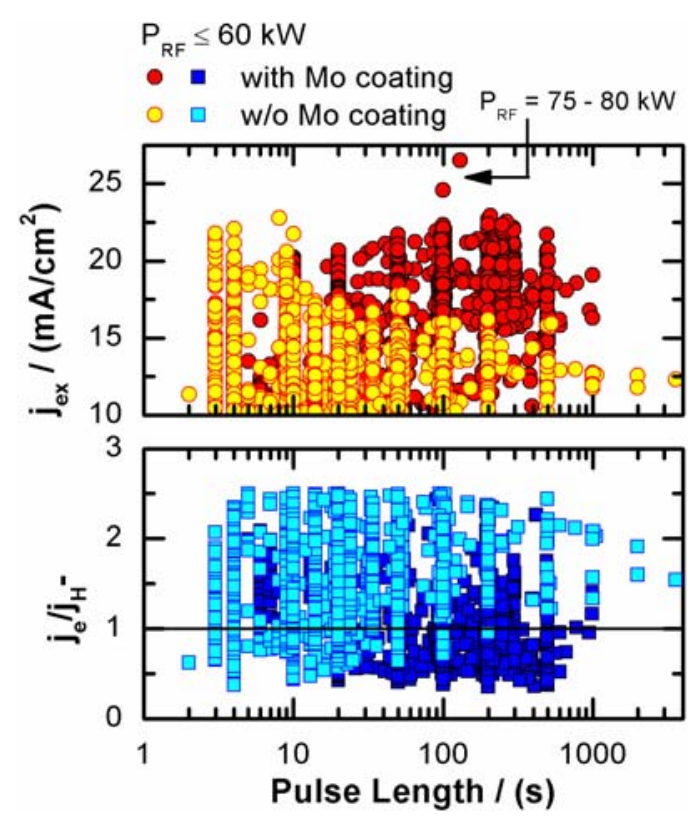

FIGURE 4. MANITU performance with and w/o Mo coating of the source [9]. MANITU source that are heavily loaded have been coated with a thin Mo layer, because there was an indication that sputtered $\mathrm{Cu}$ deteriorated the source performance by burying the Cs. First experiments with the Mo coated source surfaces showed indeed almost no copper impurities in the plasma. This is consistent with the sputtering yields: For $100 \mathrm{eV}$ - most probable the upper limit of the energy the ions can gain due to sheath acceleration at the plasma edge in the faraday screen - the self sputtering yield of copper is almost a factor 10 larger than the respective values for tungsten or molybdenum [20]. Furthermore, the sputtering threshold of copper for hydrogen/deuterium is much lower than for tungsten and molybdenum. Hence, an avalanche effect with exponentially increasing impurity content in the source can be expected for copper.

The performance of MANITU increased drastically with the coated source walls (see Figure 4 and Refs. $[9,16]$ ): stable long pulses in hydrogen and deuterium for up to $10 \mathrm{~min}$ with respect to ion and electron currents have been achieved up to now with an electron/ion ratio well below 1 and extracted current densities just below the required parameters, the latter limited by the available RF power. Hence, the inner surfaces of the ELISE source - and accordingly the ITER source [15] — will be coated with Mo.

\section{Extraction System}

The extraction system for ELISE is a three grid system - plasma grid, extraction grid and grounded grid - which will allow acceleration up to $60 \mathrm{kV}$. The source is at high potential, while the calorimeter inside the vacuum chamber is at ground potential. The aim of the design was an extraction system as close as possible to the ITER design; it has however some modifications mainly for better diagnostic access for an improved understanding of the physical processes inside the source and of negative ions extraction and for an improved cooling and filter field homogeneity. 
The overall size of the grid segments is about the same as the ITER grid segments, but in contrast to ITER, the segments are flat. The position and the size of the beamlet groups are identical to the SINGAP geometry (the MAMUG geometry is slightly different). All grids are mounted on grid holder boxes which are nested inside each other similar to positive ion PINI design. Furthermore, the grids are immersed inside the source body (see Figure 2). This design enables an absolutely flat surface of the plasma grid and allows good diagnostic access from all sides just above the grid surface.

All grids and the bias plate are electrically insulated against each other as well as against the source body to allow biasing of the source against the plasma grid and to measure electrically all grid currents. In order to have some indication of the vertical source homogeneity, the two segments of the extraction grid are also insulated against each other so that the (electron) current on these segments can be measured individually.

Table 2 shows also the expected power loads to the grids. These values are based on measurements at the IPP test facilities. The power load to the plasma grid is rather low $\left(20 \mathrm{~kW} / \mathrm{m}^{2}\right.$ at maximum) due to the effective screening of the plasma, i.e. reduction of electron density and temperature by the filter field and the plasma grid bias [12]. The total power load of the grounded grid is typically $10 \%$ of the total beam power, most probably due to back streaming ions or stripping losses; direct beam interception is not very likely due to the large diameter of the grounded grid apertures [15].

The most critical component for the source operation and also for the performance is the extraction grid. This is demonstrated in Figure 5 showing experimental data of BATMAN. Especially for deuterium with a much larger electron/ion ratio compared to hydrogen and a higher required extraction voltage, the power load of the extraction grid is much larger compared to hydrogen, both during conditioning phases and high performance operation. As can be seen in Figure 5, the interlock limit of $25 \mathrm{~kW}$ (about $200 \mathrm{~W} / \mathrm{aperture}$ ) is frequently reached for deuterium. As this value corresponds to 
roughly twice the nominal power, the maximum total power and power density for the ELISE extraction grid design was increased accordingly by an improved cooling scheme, in order to have some safety margin and a larger operating window during conditioning phases.

A good source performance and hence high current densities were obtained on BATMAN and MANITU at elevated source wall temperatures of about $40{ }^{\circ} \mathrm{C}$. In order to have some margin, the cooling system of ELISE is considered to operate between room temperature and $60{ }^{\circ} \mathrm{C}$. The water inlet operation temperature of the source and the grid system will be $55^{\circ} \mathrm{C}$ according to the actual ITER reference design. The position of the plasma grid apertures is machined with a pre-offset taking into account the different operating temperatures of the extraction/grounded grid (55 ${ }^{\circ} \mathrm{C}$ ) and the plasma grid $\left(150^{\circ} \mathrm{C}\right)$. For that case all apertures will be in line, steering by aperture offset is not planned on ELISE. The remaining expansion for the operating range of $\pm 50^{\circ} \mathrm{C}$ of the plasma grid causes a maximum aperture offset for the outer most apertures of $0.17 \mathrm{~mm}$ in vertical direction and $0.56 \mathrm{~mm}$ in the horizontal direction. The effect on beamlet deflection has been calculated by KOBRA-3D and was found to be just acceptable [21]. The worst case is for the orthogonal orientation of the electron suppression field (see below) to the filter field and the outermost apertures: here the beam just touches the wall of the extraction grid.

\section{Optimum Magnetic Filter Field Configuration}

The filter field of the reference design of ITER consists of a combination of a $4 \mathrm{kA}$ plasma grid current and permanent magnets outside the source. This filter field was optimized for minimum electron and ion deflection in the accelerator and to ensure the necessary $\int B_{x} d z$ for electron suppression for deuterium operation. For both types of small sources (filamented source and the BATMAN IPP RF source) a typical value of 0.9 to $1.1 \mathrm{mTm}$ was sufficient to achieve the ITER relevant parameters (see Figure

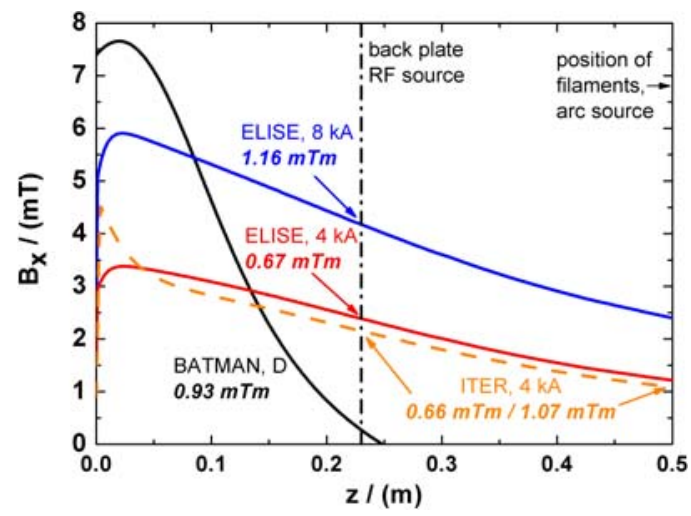

Figure 6: Filter field strength for different combinations of permanent magnets $(\mathrm{CoSm}$, $30 \times 20 \mathrm{~mm}^{2}$ ) and plasma grid currents and the corresponding values for $\int B_{x} d z$ for the RF source and the filamented source, respectively. Also shown for comparison is the value for

BATMAN for deuterium operation. $6)$.

The reduced depth of the RF source, however, has consequences for the value of $\int B_{x} d z$. By using the reference filter field (permanent magnets and $4 \mathrm{kA}$ of plasma grid current) for the ITER RF source, the $\int B_{x} d z$ is reduced by a factor of about 1.5 compared to the optimum value for BATMAN and the filamented source (see Figure 6). To obtain the optimum value, the plasma grid current has to be increased accordingly with all the consequences for electron and ion deflection in the accelerator. Hence, in order to have some operational margin, the ELISE plasma grid was designed for $8 \mathrm{kA}$ maximum current. 
The magnetic filter field in a negative source reduces the co-extracted electron current, but also bents the negative ions that are accelerated by the sheath potential from the plasma grid into the source back to the apertures. However, it is still not clear what the really important parameter is: the $\int B_{x} d z$, the filter field strength near the plasma grid, or the 3-dimensional structure of the field. Additionally, the electron deflection field from the extraction grid reaches also into the source and leads to further complexity. There is evidence from BATMAN that this field is necessary for a sufficient suppression of the co-extracted electrons. Hence, the design of the extraction grid was made flexible so that the electron suppression magnets can be installed with their respective magnetic field perpendicular (as in BATMAN and MANITU) or parallel to the filter field. The latter case was chosen for ITER in order to minimize horizontal beam deflection; however, zero field regions in front of the plasma grid are created [14] with unknown consequences for the local electron suppression.

Furthermore, due to the increase of the ELISE source size, the horizontal distance of the permanent magnets is larger as it is in the ITER reference field. This results in a reduced field in the centre of the source; the increase of the field towards the outermost apertures is less pronounced, however. This may be an advantage for the homogeneity of the beam. The experience at MANITU with different aperture patterns showed indeed, that the more apertures have been in the region of the increasing filter field, the less beamlets could be accelerated to the calorimeter [22].

Due to the apertures and the beamlet group arrangement of the plasma grid, the current distribution and hence the resulting filter field cannot be uniform. It is not clear up to now whether this is harmless for the plasma homogeneity, but it has certainly consequences for the electron and ion deflection in the accelerator. Hence the design of the plasma and the extraction grid was optimized with an emphasis on a homogeneous temperature of the plasma grid surface - to minimize the risk of inhomogeneous Cs coverage - as well as on a current distribution on the plasma grid as homogeneous as possible. The latter was achieved by optimizing the current density distribution on the plasma grid by introducing pockets into the grid so that the current is forced to flow around the apertures (for details see Ref. [14]). Figure 7 shows the difference of the new ELISE design to the ITER design: the magnetic field ripple is much more reduced; in $20 \mathrm{~mm}$ distance from the plasma grid, the magnetic filter field is almost flat.

The issue of the optimum fields in the source - and in the extraction grid - will be certainly a main topic for ELISE as well for the ongoing modeling and experimental efforts at IPP. The flexibility of ELISE with access to the source near the plasma grid - allows an easy change of permanent magnets outside the source as well of other means of creating a sufficient, simple local filter field without an appreciable field in the accelerator like the plasma grid current solution. Examples may be magnets placed in the plasma grid (pockets) or to introduce

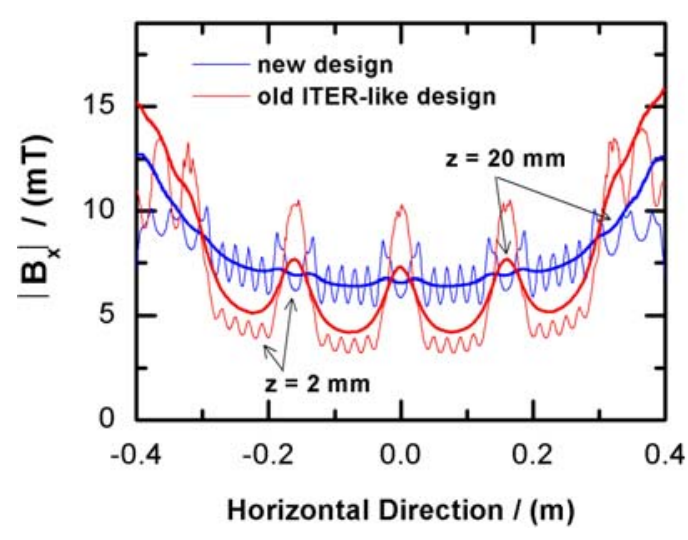

FIGURE 7. Magnetic filter field of ELISE for $8 \mathrm{kA}$ plasma grid current at different distances from the plasma grid for the ELISE design and the ITER design, respectively. 
vertical magnet rods between the aperture groups.

\section{Calorimeter}

A first conceptual design of a full power calorimeter has been started. Figure 8 shows the calculated power density profile in a distance of $3 \mathrm{~m}$ from the grounded grid for a full beam and a beamlet divergence of 2 degree. Even for this rather high divergence the upper and the lower half of the beam are clearly separated due to the large gap between the grid segments $(96 \mathrm{~mm})$ and due to the fact that no steering nor focusing is done in the ELISE grids.

The maximum power density is rather low, being about $3 \mathrm{MW} / \mathrm{m}^{2}$ in the center of the segment. It increases to about 3.6 MW $/ \mathrm{m}^{2}$ for 1 degree divergence. This

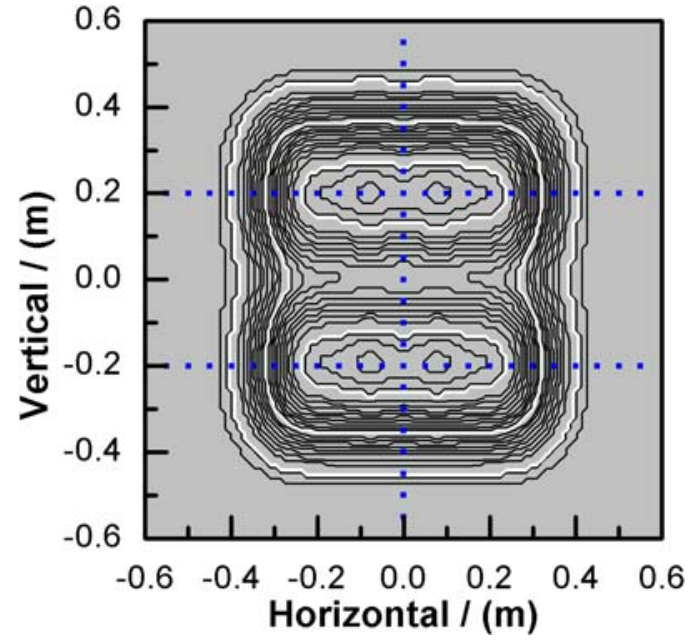

FIGURE 8. ELISE beam profile in a distance of $3 \mathrm{~m}$ for a total power of $1.2 \mathrm{MW}$ and a divergence of 2 degree. The maximum power density is $2.9 \mathrm{MW} / \mathrm{m}^{2}$. The white lines indicate the $10 \%, 50 \%$ and $90 \%$ curvatures, respectively. Also indicated is a possible distribution of TC's for beam profile measurements. together with the pulsed beam gives the opportunity to use an inertially cooled calorimeter, i.e. a copper plate with a cooling circuit at the back of the plate. The temperature increase of a $2 \mathrm{~cm}$ thick copper plate for a $10 \mathrm{~s}$ pulse with that power density is about $450 \mathrm{~K}$, which is sufficiently low. A cooling loop with about $10 \mathrm{l} / \mathrm{min}$ only is sufficient to cool down the calorimeter within the $160 \mathrm{~s}$ before the next $10 \mathrm{~s}$ pulse.

The separation of the upper and lower half of the segments can be used for beam profile measurements. A possible arrangement of thermocouples is also shown in Figure 8. The thermocouples can be embedded in almost thermally isolated areas of the copper plate with a low thermal contact to the main plate, as it is now the case for the BATMAN calorimeter. These areas can consist of copper or graphite for thermography from the backside.

\section{Diagnostic Setup}

The signals/diagnostics at the test facilities in IPP are divided in three groups: (1) control parameters for protection and safe operation, as well as for operational parameters; examples are voltages, currents, temperatures, RF power; but also more complicated quantities like the power on the extraction grid, calculated on-line by the control system; (2) performance parameters for the source conditioning status and the source and beam 'quality'; examples are the electron/ion ratio, the calorimetric current density, source and beam homogeneity, beam divergence, Cs content; (3) physics parameters for better understanding of the processes in the source and as input for modeling; examples are the electron density and temperatures or the negative ion density.

The main diagnostics - the "work horses" - are the measurement of the currents at the various grids and in the HV system, the calorimeter and the optical emission spectroscopy both in the source and the beam. A special feature of the ELISE design is 
that the two segments of the extraction grid are insulated against each other and the grid holder box (see above) so that the current on each segment can be measured separately. Hence, a rough evidence of the top-bottom source homogeneity can be obtained.

In order to measure the Cs dynamics also in between the discharges (i.e. the gas or vacuum distribution), Cs absorption spectroscopy is foreseen. This was already successfully tested at the University of Augsburg [23]. The use (if or how) of the other physical diagnostics (Langmuir Probes, Laser Detachment and Cavity Ringdown spectroscopy) is still not decided; all three are problematic due to space limitations (for the probes) due to the small HV insulation distances and due to their complexity.

\section{CONCLUSIONS}

ELISE is an important intermediate step for the RF source development for the ITER NBI system. With respect to ISTF/NBTF, the ELISE design offers a larger experimental flexibility for the investigation of concept improvements and a better diagnostic access to the extraction region near the plasma grid.

\section{ACKNOWLEDGEMENTS}

The work was (partly) supported by a grant (\#03FUS0002) from the German Bundesministerium für Bildung und Forschung as well as by a grant (\#TW6-THHNRSFD4) from the European Union within the framework of EFDA (European Fusion Development Agreement). The authors are solely responsible for the content.

\section{REFERENCES}

1. ITER EDA Documentation Series No. 24, Plant Description Document, Sec. 2.5.1. IAEA 2002

2. E. Speth et al. Plasma Science and Technology 6 (2004) 2135

3. E. Speth et al., Nuclear Fusion 46(6) (2006) S220

4. P. Franzen et al., Nuclear Fusion 47 (2007) 264

5. A. Stäbler et al., Symposium on Fusion Technology, 2008

6. R. Hemsworth et al., Review of Scientific Instruments, vol.79, no.2, Feb. 2008, pp. 02C109-1-5

7. B. Schunke et al., these proceedings

8. W. Kraus et al., Review of Scientific Instruments, vol.79, no.2, Feb. 2008, pp. 02C108-1-3

9. W. Kraus, Proceedings of the $35^{\text {nd }}$ EPS Conference, 2008

10. P. Franzen et al., Fusion Engineering and Design 82 (2007) 407

11. U. Fantz et al., Review of Scientific Instruments, vol.79, no.2, Feb. 2008, pp. 02A511-1-6

12. U. Fantz et al., these proceedings

13. B. Heinemann et al., Symposium on Fusion Technology, 2008

14. R. Nocentini et al., Symposium on Fusion Technology, 2008

15. D. Marcuzzi et al., Symposium on Fusion Technology, 2008

16. W. Kraus et al., these proceedings

17. R. Hemsworth et al., AIP Conference Proceedings 925 (2007) 290

18. A. Lorenz et al., Technical Report IPP 4/285, Max-Planck-Institut für Plasmaphysik, Garching, 2006

19. P. Franzen et al., AIP Conference Proceedings 993 (2008) 51

20. W. Eckstein et al., Sputtering Data, IPP Report 9/82.

21. R. Gutser et al., these proceedings

22. W. Kraus et al., Fusion Engineering and Design 74 (2006) 337

23. U. Fantz et al., to be published 